\title{
Laboratory field studies of mini potato planter
}

\author{
Nikolai P. Laryushin ${ }^{1}$, Oleg N. Kukharev ${ }^{1}$, Anton S. Bochkarev ${ }^{1, *}$, and Vladimir S. Bochkarev ${ }^{2}$ \\ ${ }^{1}$ Penza State Agrarian University, Penza 440014, Russia \\ ${ }^{2}$ Mokshan Agricultural Technology College, Mokshan 442370, Penza region, Russia
}

\begin{abstract}
The paper considers the design and operation of the experimental mini potato planter for smallscale farms (private subsidiary farms - PSF). It presents the results of field studies of experimental potato planter in field conditions. The paper also describes probabilistic curves of potato tubers distribution in a furrow depending on motion speed and design parameters of tubers speed damper.
\end{abstract}

\section{Introduction}

Potatoes are one of the most important products in a human diet. It is produced in 145 countries via various technologies. Potatoes are grown for both fresh or processed food and cattle feed.

In recent years the total area of potato planting in Russia varies at the level of 2 million hectares. The yield is within $11.5 \ldots 22.5 \mathrm{t} / \mathrm{ha}$. Currently more than $80 \%$ of potatoes in our country are produced in PSF with planting areas of less than 2 hectares. At the same time, the level of mechanization in these farms is low, including potato planting. Labor costs for potato growing even in small farms are quite high [1].

Potato planting is one of the key production processes. The quality of planting depends on yield and quality of potato storage.

The analysis of potato planting makes it possible to conclude that the utilized mini potato planters for potato tubers do not fully meet the agricultural requirements due to the absence of tubers speed dampers when they are laid in a furrow.

Therefore, the improvement of the quality of potato tubers planting by developing and using a mini potato planter with a speed damper of potato tubers when laying them in a furrow seems quite relevant and practically important for agricultural production.

The use of small mechanization means in private subsidiary farms is caused by their small size and small processed areas.

\section{Materials and methods}

In order to solve the problem of potato tubers planting in PSF, an experimental mini potato planter with tuber speed damping device used when they are laid in a furrow was designed and manufactured (Fig. 1). The potato planter includes a frame, a tanker for tubers, a chain-spoon feed, a ploughshare, balancing drive wheels, covering disks and a speed damper of tubers when they are laid in a furrow.
While in operation the tubers from a tanker are supplied to a chain-spoon feed, which drops tubers from a lower part of planting unit into a furrow made by a ploughshare, and the tubers are pressed by elastic seed speed damper to a furrow bottom preventing their bounce and rolling along the furrow. The covering disks fill fixed tubers with soil, thereby maintaining a predefined step of potato tubers thus improving the uniformity of tuber distribution along the length of a furrow and the depth of seed placement, which improves the planting quality and increases potato yield.

An application for invention is submitted for this potato planter with a speed damper of tubers when they are laid in a furrow.

The experimental potato planter was studied in a test site in 2019 using an experimental mini potato planter with a developed speed damper of tubers when they are laid in a furrow using STO AIST 5.2-2010. Tests of agricultural machinery. Potato planters. Methods of functional assessment [2], GOST 28306-2018. Agricultural equipment. Potato planting machines. Test methods [3]. For laboratory field studies, Matushka potato seeds with a planting rate of $23.8 \mathrm{c} / \mathrm{ha}$ were used [4].

In order to carry out laboratory field studies of the potato planter with a developed speed damper of tubers when they are laid in a furrow, we selected an area in accordance with agricultural technical requirements and taking into account the place in crop rotation of the cultivated culture. We defined type, moisture, hardness, mechanical composition, relief and micro-relief of soil of the chosen area.

The study to determine soil moisture and hardness made it possible to define that the average soil hardness was $0.589 \mathrm{MPa}$ and the average soil moisture was $19.36 \%$. These values are necessary to determine the conditions of growth and development of plants (water, air and heat modes of soil, mechanical resistance of soil to the root system of plants).

To define dimensional and mass characteristics of tubers, the tubers, which were dug out from each

\footnotetext{
* Corresponding author: bochkarev.a.s@pgau.ru
} 
registration area, were mixed and an average sample of 200 pieces was selected. The size of potato tubers was defined via ШЦЦ-10-150.01 caliper. The caliper allows measuring up to $0.1 \mathrm{~mm}$.

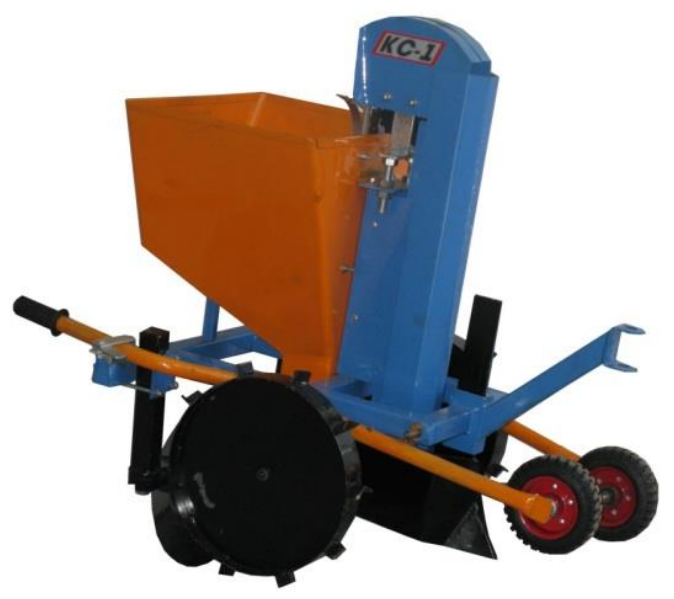

Fig. 1. Experimental mini potato planter

The potato tuber size is defined after placing a tuber on a flat surface. The tuber is measured (length) and data is recorded in the survey list. Then another measurement (width, thickness) is made and so on. The tubers were selected for testing according to GOST 12036-85. In compliance with the method of studying physical and mechanical properties of agricultural plants, the number of measurements was taken as 100 with triple repetition of the experiment.

\section{Results}

As a result of experimental data processing, histograms of potato tubers distribution by length, width and thickness were designed (Fig. 2-4).

The purpose of the experiment was to define the optimal structural and kinematic parameters of a mini potato planter [5].

The object of the study was the technological process of planting potatoes with a mini potato planter. The subject of the study included operating patterns, conditions and modes of a mini potato planter.

The results were processed using Statistica 6.0 and Excel software. The assessment criteria were as follows: uniformity of potato distribution in a furrow.

Functional indicators were determined in areas meeting the requirements of the operating instructions under specific conditions. For the functional test, measuring areas of 100 distances between tubers in a furrow were chosen at test sites.

The experimental potato planter approaches the measuring area at a nominal operating speed filled by at least 0.5 of maximum tanker capacity. The mini potato planter is adjusted to the setting planting distance, i.e. according to the planting density of 50,000 pieces \pm $10 \%$ tubers per hectare. For each speed, one (on a flat area) or two passes (straight and back on the area with a slope) were made with tankers filled with at least 0.5 of their volume.

The tanker capacity of was defined according to the volume of a planting material.

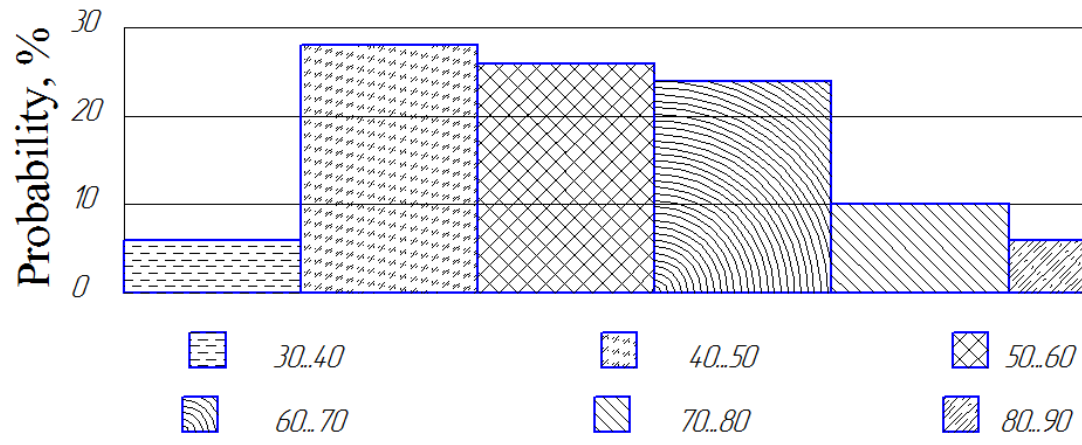

Length, $\mathrm{mm}$

Fig. 2. Histogram of Matushka potato tubers distribution by length

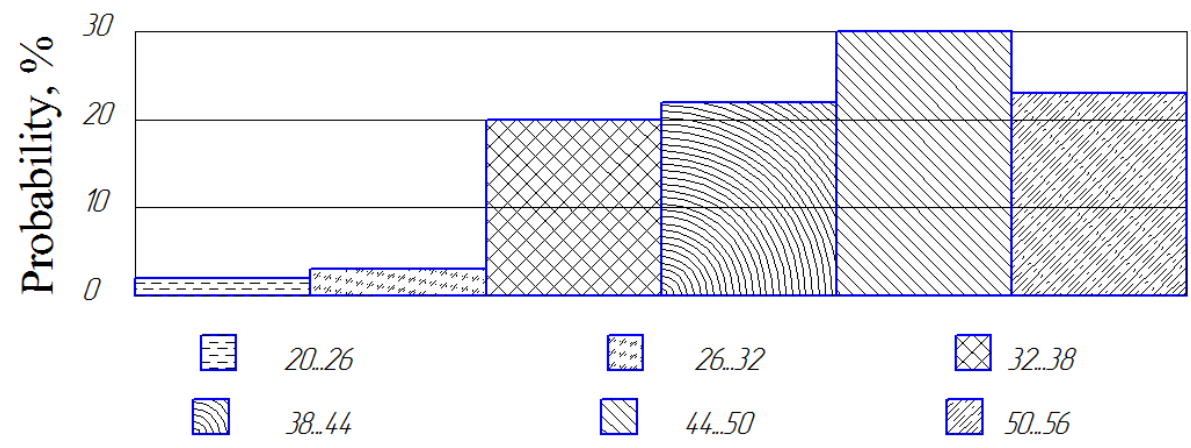

Width, mm

Fig. 3. Histogram of Matushka potato tubers distribution by width 


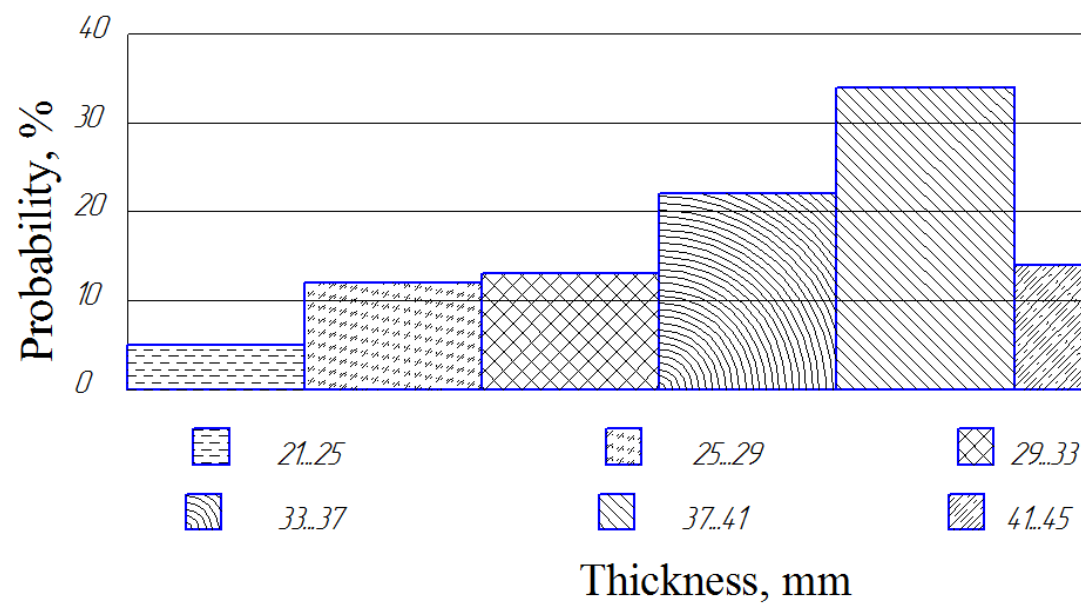

Fig. 4. Histogram of Matushka potato tubers distribution by thickness

The nominal operating speed was measured after stabilization of the required mode. No loss of controllability, violation of the technological process and conditions of safe operation of the maintenance team, deterioration of the quality of work are allowed during measurements.

The maximum speed of a mini potato planter according to GOST 28708-2001 [6] did not exceed $4.3 \mathrm{~km} / \mathrm{h}$. The speed of an elevator planting unit equaled the speed of a mini potato planter $(\lambda=1)$.

Functional indicators were determined using a calibrated planting material belonging to the same size group. Cut tubers were not used according to GOST 208306-89 [7]. Undamaged planting material is used to determine tuber damage by a machine.

The distance between tubers in a furrow in meters (centimeters) was determined at the measuring segment by measuring the distances between the centers of adjacent tubers on the longitudinal axis of the furrow (covering disks are placed up). The average actual distance between tubers in a furrow in meters (centimeters) was defined as the arithmetic mean of 100 distances between tubers in a furrow.

For each operating mode, at least 800 tuber distances were measured. A coefficient of variation was used as an additional value to express the actual distance between tubers.

Before taking the potato distribution into account, the furrows were carefully examined: tubers covered with earth were carefully opened, large earth-balls were removed from the groove, the soil surface in the furrow was leveled to obtain a more uniform tension of a tape measurer.

The actual distance between the potatoes was determined by sequential subtraction. The class interval made $5 \mathrm{~cm}$. The average distance, standard deviation, variation coefficient, number of cases with a certain interval $(0 \ldots .5,5 \ldots 10 \mathrm{~cm}$, etc.) was calculated as a percentage of the total number of measurements. According to the number of cases at certain intervals, a graph of uniformity of potato layout in a furrow was plotted. The results were processed using mathematical statistics. The measured values were rounded up to a whole number.

The number of single potatoes, twins and passes as a percentage of the total number of measurements was counted in the analysis. According to the actual distance between potato centers we defined the uniformity of potato spacing in a furrow ( \pm 0.2 from an average).

The uniform distribution of tubers $(\mathrm{R})$ in percentage points was defined by the formula:

$$
R=\frac{t}{n} \cdot 100,
$$

where $\mathrm{t}$ - number of landed tubers, between which the actual distance makes from 0.8 to 1.2 of adjusting distances between tubers in a furrow $(( \pm 0.2$ from an average);

$\mathrm{n}$ - total number of tubers planted in a measuring area with the setting distance between tubers in furrow A.

The percentage of passes (M) was determined by the formula:

$$
M=\frac{j}{n} \cdot 100,
$$

where $\mathrm{j}$ - number of passes.

The percentage of twins (D) was determined by the formula:

$$
D=\frac{k}{n} \cdot 100,
$$

where $\mathrm{k}$ - number of twins.

The planting error $(\mathrm{K})$ in percentage points was determined by the formula:

$$
K=\frac{j+k}{n} \cdot 100,
$$

The retention factor between tubers in a furrow (R) was determined by the formula:

$$
R=\frac{A_{n}}{A_{t}} \cdot 100,
$$

where $A_{t}-$ average distance between tubers in a furrow.

Lateral deviation of tubers in a furrow in meters (centimeters) is determined by measuring this deviation for each planting device from the perpendicular passing through the longitudinal axis of a furrow (a furrow coverer does not work). 
As a result of processing, the share of tubers with deviation above permissible value is calculated.

The frequency of tubers planting $(F)$ per second is determined by formula

$$
R=\frac{V_{y}}{A_{t}},
$$

where $V_{y}$ - nominal operating speed, $\mathrm{m} / \mathrm{s}$.

The planting density $(\mathrm{H})$ of tubers per hectare was determined by the formula:

$$
F_{p}=\frac{10^{8}}{A_{K} \cdot B}
$$

where $\mathrm{B}$ - width between furrows, $\mathrm{cm}$;

$10^{8}$ - area of 1 hectare of $\mathrm{cm}^{2}$.

The consumption of the planting material $(G)$ in tons per hectare was determined by the formula:

$$
G=H \cdot m \cdot 10^{-6},
$$

where $m$-average mass of tubers, g.

The depth of tuber drilling $\mathrm{h}_{\mathrm{d}}$ in centimeters was determined for each furrow of tubers. The perpendicular distance from the bottom edge of a tuber to the field surface before planting was measured (a furrow coverer does not work, the resulting ridges are leveled). The result is the arithmetic mean of not less than 30 measurements made uniformly over the whole plate of the measuring section. Measured minimum and maximum drilling depths are also recorded in the protocol.

The depth of soil loosening under the tuber $\mathrm{P}$ in centimeters was determined by distance measurements. The distance is measured along perpendicular from the lower edge of a tuber to untreated soil under the tuber (at furrow bottom).

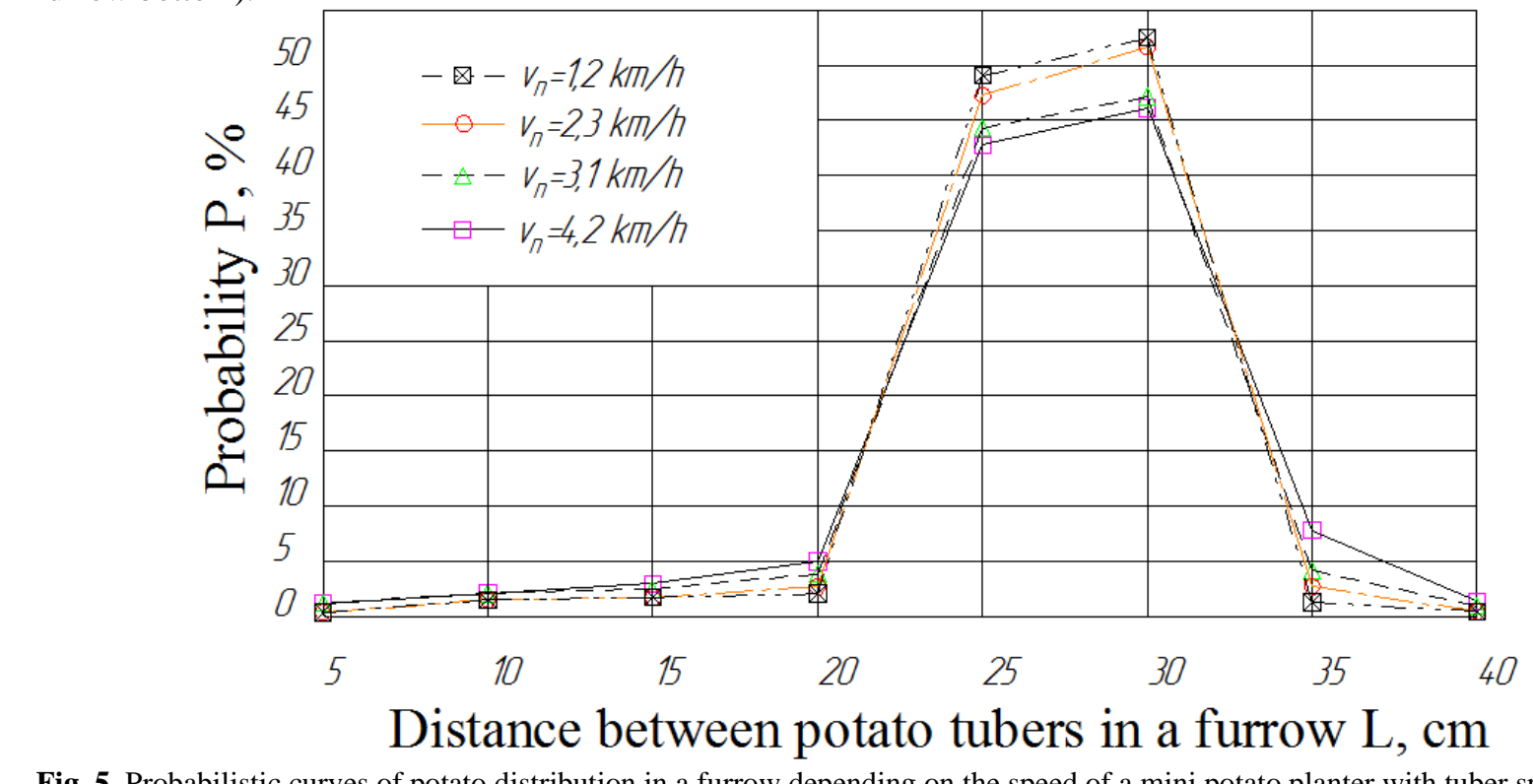

Fig. 5. Probabilistic curves of potato distribution in a furrow depending on the speed of a mini potato planter with tuber speed

damping device used when they are laid in a furrow
The result is the arithmetic mean of 30 measurements made uniformly over the entire area of the measurement section.

Tuber damage as a percentage was determined for 100 tubers planted in each furrow. Planted tubers were classified while damaged into the following groups:

1) without damage:

2) slight damage - damage depth up to $1.7 \mathrm{~mm}$;

3) mean damage - depth of damage from 1.7 to $5 \mathrm{~mm}$;

4) severe damage - damage depth more than $5 \mathrm{~mm}$.

The result is the share of tubers in selected groups from the total number of planted tubers in percentage.

The number of single root crops, twins and passes as a percentage of the total number of measurements was counted in the analysis. According to the actual distance between potato centers we defined the uniformity of potato distribution in a furrow ( \pm 0.2 from an average).

According to experimental data, probabilistic curves of potato distribution along the furrow were built depending on the speed of the elevator-type planting unit - v (Fig. 5).

By analyzing the curves in Figure 5 it can be concluded that in the movement range of a mini potato planter from 1 to $4 \mathrm{~km} / \mathrm{h}$, the number of normal intervals $(\mathrm{M} \pm 0.2 \mathrm{M})$ decreases from 95 to $87 \%$. At the same time the variation coefficient $(v)$ changes from 17.20 to $22.14 \%$, the average value increases from 23.55 to $24.74 \mathrm{~cm}$, the mean square deviation $(\sigma)-$ from 3.68 to $4.45 \mathrm{~cm}$, the number of passes increases from 2 to $12 \%$. The amount of slightly damaged tubers varies within 1.5...6.2\%, severe damage is not observed. The planting error (K) (Figure 6) increases from 2.2 to $9.7 \%$ depending on the speed of a mini potato planter. 


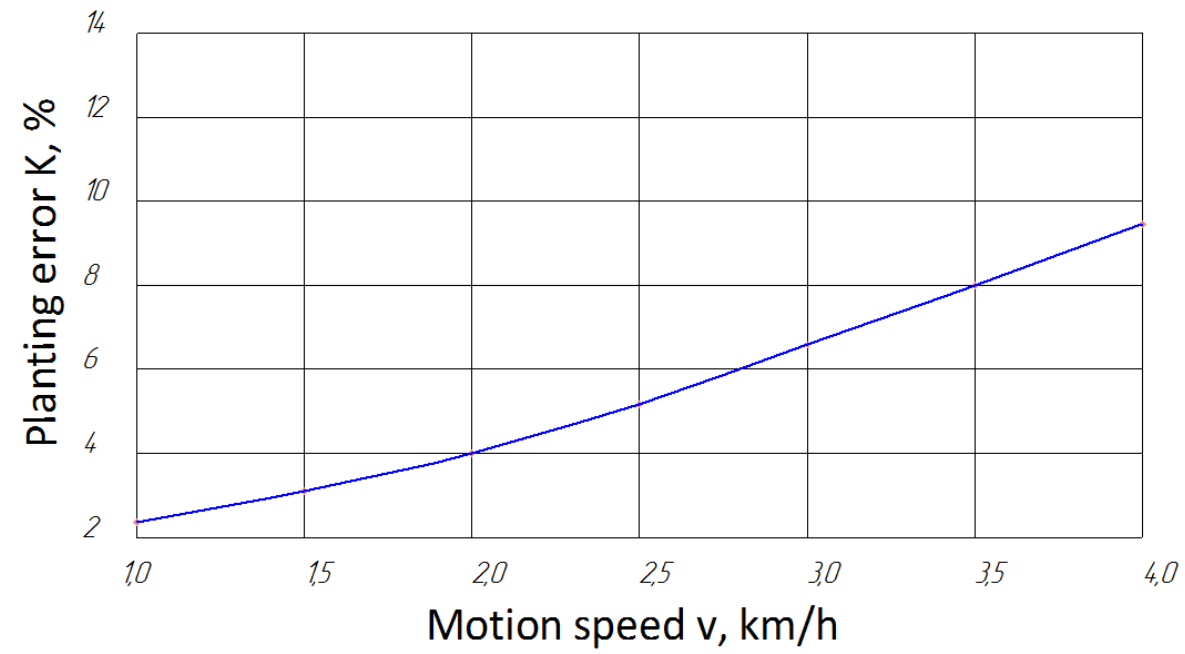

Fig. 6. Dependence of the planting error on the speed of a mini potato planter with tuber speed damping device used when they are laid in a furrow

Figure 5 also shows that the optimal motion speed of a mini potato planter will be $\mathrm{v}=1.2 \mathrm{~km} / \mathrm{h}$, at which the number of normal intervals $\mathrm{M} \pm 0.2 \mathrm{M}$ equals $95 \%$, at the same time up to the speed of $4.3 \mathrm{~km} / \mathrm{h}$ the machine operates in compliance with agricultural requirements in terms of the accuracy of potato layout.

Labor productivity during potato planting makes up to $0.15 \mathrm{ha} / \mathrm{h}$ of the net time. The use of a mini potato planter in private farms allows increasing labor productivity up to 2.5 times [8].

\section{Conclusion}

The study showed that the mini potato planter with tuber speed damping device used when they are laid in a furrow operates in accordance with agricultural requirements at a speed of up to $4.3 \mathrm{~km} / \mathrm{h}$.

\section{References}

1. N.N. Kolchin, K.A. Pshenichnikov, S.B. Priamov, Russia's accession to the WTO: problems and prospects of Russian potato growing, Potat. and vegetabl., 7, 2-3 (2012)
2. STO AIST 5.2-2010. Tests of agricultural machinery, Potato planters, Methods of functional assessment

3. GOST 28306-2018. Agricultural machinery. Potato planting machines. Test methods

4. E.A. Simakov, B.V. Anisimov, A.V. Korshunov et al., Varietal resources and best practices of potato production (Rosinformagrotech, Moscow, 2005)

5. E. A. Simakov, Potato varieties cultivated in Russia: 2010, Annual reference publication (Agrospas, Moscow, 2010)

6. GOST 28708-2001. Means of small-scale mechanization of agricultural works, Safety requirements (Publishing of Standard, Minsk, 2003)

7. GOST 28306-89. Potato planting machines, Test methods (Standardtinorm, Moscow, 2006)

8. N.P. Laryushin, K.Z. Kukhmazov, A.V. Polikanov, O.N. Kukharev, Economic assessment of crop production technologies (on the example of seed onion production) (Penza, 2001) 